\title{
Evaluating the English and Thai-Versions of the Psychological Well-Being Scale across Four Samples
}

\author{
Piyanee Klainin-Yobas ${ }^{1 *}$, Wareerat Thanoi ${ }^{2}$, Nopporn Vongsirimas ${ }^{2}$, Ying Lau ${ }^{1}$ \\ ${ }^{1}$ Alice Lee Centre for Nursing Studies, National University of Singapore, Singapore \\ ${ }^{2}$ Department of Mental Health and Psychiatric Nursing, Faculty of Nursing, Mahidol University, \\ Bangkok, Thailand \\ Email:^nurpk@nus.edu.sg,wareerat.tha@mahidol.ac.th,nopporn.von@mahidol.ac.th,nurly@nus.edu.sg
}

How to cite this paper: Klainin-Yobas, P., Thanoi, W., Vongsirimas, N., \& Lau, Y. (2020). Evaluating the English and ThaiVersions of the Psychological Well-Being Scale across Four Samples. Psychology, 11, 71-86.

https://doi.org/10.4236/psych.2020.111006

Received: December 6, 2019

Accepted: January 5, 2020

Published: January 8, 2020

Copyright $\odot 2020$ by author(s) and Scientific Research Publishing Inc. This work is licensed under the Creative Commons Attribution International License (CC BY 4.0).

http://creativecommons.org/licenses/by/4.0/

\begin{abstract}
Psychological well-being is an important predictor for various health outcomes. This study aimed to test psychometric properties of the English- and Thai-version of the Psychological Well-Being Scale (PWB-S). Four convenience samples were recruited in Singapore and Thailand; and the English and Thai-version of the PWB-S were used. Data collection included paper-andpencil and online self-reported questionnaires. Descriptive statistics and exploratory and confirmatory factor analyses were performed to test the factorial structure of the scale. Correlations with external variable were utilized to test convergent validity. For all four samples, the PWB-S contained two distinct factors: The Autonomy \& Growth (9 - 10 items) and Negative Triad factors (5 - 6 items). All four samples had acceptable fit indices and all factor loadings achieved statistical significance. All factors had significant correlations with external correlates and reliability coefficients were acceptable. Findings suggested that the English and Thai-version PWB-S had sound psychometric properties for Thai and Singaporean samples. Future research may use the English and Thai-version of PWB-S to assess psychological wellbeing among young people.
\end{abstract}

\section{Keywords}

Psychological Well-Being, Construct Validity, Students, Singapore, Thailand

\section{Introduction}

Psychological well-being (PWB) is one of the important concepts in positive 
psychology or positive mental health. PWB reflects not only the absence of mental problems but also the presence of positive psychology including subjective wellbeing (such as positive effect, life satisfaction and happiness) (Diener, 1984) and eudaimonic well-being (autonomy, environmental mastery, purpose in life, personal growth, positive relations and self-acceptance) (Ryan \& Deci, 2001). The recent paradigm shift has focused on promoting physical and psychological health and several studies were conducted to support such trend. A literature review highlighted that PWB was related to lowered incidence of health problems (including heart problems, stroke, venous thromboembolism, hypertension, lung diseases, arthritis, diabetes, and cancer) and slow progression of cardiovascular diseases and decreased mortality (Hernandez et al., 2018). PWB is also linked with biological outcomes. Specifically, people with high levels of eudaimonic well-being had lower levels of daily salivary cortisol, pro-inflammatory cytokines, cardiovascular risks and longer duration of REM sleep (Ryff, 2014; Ryff, Singer, \& Love, 2004). PWB is an important indicator for positive affective states, optimal functioning and social life (Ryff, 1995; Ryff \& Singer, 1996; Ryan \& Deci, 2001). An ability to manage negative emotions occurring in life are essential for long-term well-being (Ryff, 1989a; Ryan \& Deci, 2001; Liu, Shono, \& Kitamura, 2009). On contrary, decreased PWB was associated with increased depression and anxiety among people with diabetes (Ramkisson, Pillay, \& Sartorius, 2016). Given the importance of the PWB in predicting other health outcomes, it is essential to promote PWB among people with various sociocultural backgrounds such as adolescents and young adults.

Young adults encountered various issues that may negatively impact their psychological well-being. Young adults refer to those aged from 10 to 24 years (World Health Organization (WHO), 2014) and their developmental tasks include accepting physical changes, acquiring skills for adult relationships, developing abstract thinking, achieving emotional independence and preparing for their future career (Paperny, 2011). Some adolescents are able to accomplish the tasks smoothly; however, the others may encounter challenges and experience the feelings of vulnerability and insecurity. Sociocultural contexts; and support from family, school, and community are of great importance to facilitate adaptation and transition from adolescents to adulthood (World Health Organization (WHO), 2011). Ineffective adaptation to the changes and inability to fulfill development tasks in adolescents may attribute to the onset of several mental disorders such as depression, anxiety, substance abuse, aggression and violence, conduct disorder, and self-harm behavior (Stuart, 2009). There is a need to prevent such mental disorders and promote PWB among young adults. A sound screening tool is also required to assess PWB in this population.

\subsection{The Psychological Well-Being Scale (PWB-S)}

Guided by the psychological well-being model (Ryff, 1989a, 1989b), the Ryff's psychological well-being scale (PWB-S) was developed to capture the concept of PWB. The original PWB-S entailed 120 items, comprising six dimensions and 
the scale has been generally used in several groups and several countries such as American and Canadian (Kafka \& Kozma, 2002; Ryff, 1989a; Ryff \& Keyes, 1995). The six dimensions assess different challenges individuals face in an effort to function optimally and they include self-acceptance (SCC), positive relations with others (RELAT), environmental mastery (ENV), autonomy (AUTO), purpose in life (PUR), and personal growth (GROW). The scale was tested for psychometric properties and reported with Cronbach's a ranging from 0.86 to 0.93 (Ryff, 1989a, 1989b). Test-retest reliability coefficients ranged from 0.81 to 0.88 over a duration of six weeks (Ryff, 1989a, 1989b). However, the factor structure of the 120-item PWB-S was not supported by any statistical tests (such as factor analyses). In 1994, the 84-item PWB-S was devised and some original items were removed based on results from item-total correlations (Ryff, Lee, Essex, \& Schmutte, 1994). Correlation coefficients between the original and shorted version were in the range of $0.97-0.98$ (Ryff et al., 1994). However, factor analyses were not performed on the 84-item PWB-S and thus its factor structure remains unclear (Ryff et al., 1994). In 1995, Ryff and Keyes revised the original 120-item scale by selecting three out of 20 items from each dimension, making the 18 -item PWB-S.

\subsection{Validations of the PWB-S in Different Samples}

Different versions of the PWB-S were tested in different countries such as American, Canadian, and British samples (Abbott, Ploubidis, Huppert, Kuh, Wadsworth, \& Croudace, 2006; Clarke, Marshall, Ryff, \& Wheaton, 2001; Kafka \& Kozma, 2002; Keyes, Shmotkin, \& Ryff, 2002; Marmot, Ryff, Bumpass, Shipley, \& Marks, 1997; Ryff, 1989b; Ryff \& Keyes, 1995; Ryff, Keyes, \& Hughes, 2003; Ryff \& Singer, 2006; Van Dierendonck, 2004). It is important to test psychometric properties of the PWB-S because it has been widely used and cited among researchers. Ryff \& Keyes (1995) tested the 18-item PWB-S on a large national database of midlife adults. Results from confirmatory factor analyses (CFA) showed that the 18-item PWB-S had an acceptable model-fit for both the sixfactor model and second-order six-factor models (Ryff \& Keyes, 1995). This version also exhibited convergent and discriminant validity; and reliability (Ryff \& Keyes, 1995). Clarke et al. (2001) tested the multidimensionality of the 18-item PWB-S on Canadian older adults $(n=4266)$ using CFA and they reported six underlying constructs, echoing Ryff \& Keyes's (1995) findings. However, four items loaded more than one underlying factor, which suggested that such items might not be unique measurements of the factor they were supposed to capture. In 2006, Springer and Hauser tested the 18-item PWB-S on three large databases (university graduates, non-institutionalized adults and national samples) of USA. The six-factor structure was the best-fitting model; however, there were large correlations among the factors with most correlation coefficients ranging from 0.70 to 0.98. Among which, SCC were highly correlated with AUTO, RELAT, ENV, GROW, and PUR. Given the results, it is questionable if there are 
overlapping contents among the scale items or if the scale really contains six factors.

\subsection{Gaps in Existing Validation Studies}

Most studies examining the PWB-S used the English version in Western countries. Consequently, it is uncertain if the findings are applicable to the translated versions (such as the Thai version) and to other samples, especially in Asian countries (such as Singapore and Thailand). Note that the Western populations are known in terms of more individualistic values, more independent life styles and freedom, and greater importance to self-fulfillment (Faudzi, Armitage, Bryant, \& Brown, 2018). On contrary, Asian cultures portray more collectivistic values, greater intergenerational relationships and greater attachment to conservative practice (Faudzi et al., 2018). Ryff (1995) reported evidence from a cross-cultural study in that American adults rated themselves the highest on the personal growth and autonomy components of PWB-S whereas Korean adults rated the highest positive relations with others and lowest self-acceptance. There is a need to further explore the cross-cultural differences relating to the PWB-S.

\subsection{The Current Study}

The primary aim of this study was to compare the psychometric properties of the English and Thai-version PWB-S (Ryff \& Keyes, 1995) across four youth samples. Sample 1 and 2 was Thai university students Time 1 and Time 2 respectively. Sample 3 was Thai secondary school students and sample 4 was Singaporean university students. Findings from this study could provide solid evidence to support the construct and convergent validity of the measurement across countries and time.

\section{Method}

\subsection{Research Design}

We utilised comparative descriptive research design, which estimate differences in variables across two or more units of study that take place in natural phenomena (Grove, Burbs, \& Gray, 2013). The research design enabled the test and comparison of psychometric properties of the scale, including validity, factorial structure, and reliability across samples and time.

\subsection{Samples}

The target population included young adults aged $10-24$ years from two countries, Thailand and Singapore. The best settings to recruit such young adults would be secondary schools and university. Convenience sampling was used to recruit four samples. The convenience sample, using a non-probability technique, generally comprises people who are available at a given place and time slot (Grove et al., 2013). In this study, students who were readily accessible and 
available during data collection period were recruited. To enhance the generalizability of the research findings, we collected data from four samples. Sample 1 and 2 were university students and Sample 3 was secondary school students in Thailand. Sample 4 was university students in Singapore. Details of the sample are listed below.

\section{Sample 1 and sample 2}

Sample 1 and Sample 2 were undergraduate university students recruited from a public university in Thailand. Participants would be eligible for this study if they were enrolled full-time for undergraduate programs regardless of schools/faculty. All students would be excluded if they had physical and mental conditions requiring hospitalization. Following the approval from the university ethic committee (IRB), the researchers seek permission from all schools/faculties to collect data from their respective students. Then, the researchers organized out-of-class meetings with students, provided the information about the study, distributed personal information sheet (PIS) and invited them to partake in the study. Interested participants were asked to sign a consent form and complete self-reported, paper-and-pencil questionnaire. Such information contributed to Time 1 data. One year later, the same process was repeated and the Time 2 data were collected.

\section{Sample 3}

Sample 3 encompassed secondary school students recruited from three schools in Bangkok and Nakonprathom provinces, Thailand. One school had only male students, one had only female students and another one had both male and female students. Following the ethics approval, the researchers liaised with the school principals and teachers to collect data. First, the researchers arranged out-of-class meetings with students to explain the study, distribute PIS, answer questionnaires they might have and seek their participations. Then, the students were asked to bring the PIS to discuss with their parents. Interested students and their parents were then asked to sign consent and assent forms respectively. Next, another meeting was scheduled and interested students were asked to complete the self-reported, paper-and-pencil questionnaires.

\section{Sample 4}

Sample 4 included full-time undergraduate students from a university in Singapore. Inclusion and exclusion criteria were the same as those of Sample 1 and 2. The study commenced following the approval of university ethics committee. Unlike the sample 1,2, and 3, data for this sample were collected via online self-reported questionnaires. First, the researchers seek permission from the university president to collect data from students. Then, an invitation e-mail was sent to potential participants to explain the nature of the study and PIS was attached with the e-mail. The link to online questionnaire was also attached. Next, all interested participants were asked to go the link and complete the anonymous online questionnaire. Two reminders were sent to participants one week and three week later. Data from the questionnaires were then converted to IBM SPSS electronic file. 


\subsection{Instruments}

Self-reported and online questionnaires were used to collect data. The 18-item Ryff's Psychological Well-Being Scale (PWB-S: Ryff, 1989b) was used and this scale comprises six subscales: autonomy, environmental mastery, purpose in life, personal growth, positive relations with others, and self-acceptance. Respondents responded on one of six-point categories ranging from (1) strongly disagree to (6) strongly agree. Possible scores are in the range of 18-108, with higher scores signify better psychological well-being. Samples 4 used the English version of PWB-S English whereas Sample 1, 2 and 3 used Thai version. The back-translation using Brislin's (1986) method was used to translate the English PWB-S into Thai language. First, a nurse educator specialized in mental health nursing translated the original scale into Thai. Then, another nurse educator back-translated the Thai version into English. Next, the two versions were examined for semantic and content equivalence by native English speakers who had never seen the original version of the instrument. Finally, the research team (who are bilingual scholars) reviewed the original and Thai version to ensure the meaning of questionnaire items. Any problems were resolved through discussion among the research team.

PWB has a positive link with social support. This is study, social support was measured with the 12-item multi-dimensional scale of perceived social support (MSS; Zimet, Dahlem, Zimet, \& Farley, 1988), which comprised three subscales: support from family, support from friend and support from significant others. The three subscales served as external variables to test convergent validity of the PWB-S. Specifically, if the PWB-S really captures the underlying construct of PWB, it should manifest positive correlations with all social support subscales.

\subsection{Statistical Analysis}

For all four samples, data analyses included univariate statistics, exploratory factor analyses (EFA), confirmatory factor analyses (CFA), reliability analyses and correlational tests. First, participants' personal information was computed by using univariate statistics (such as mean, standard deviation and percentage) with IBM SPSS version 24.0. Secondly, a series of EFA were performed to identify a factorial structure of the PWB-S. We first examined the six-factor solution as suggested by the scale developer, followed by $2,3,4$, and 5 factors and compared all the results. In order to determine the number to factors to extract, we looked at both statistical (such as Eigen values and Scree plots) and substantive issues (including parsimony, interpretability of the extracted factors, theoretical sensibility of questionnaire items, and previous evidence) (Fabrigar, MacCallum, Wegener, \& Strahan, 1999). Each item of factor loadings that is greater than 0.40 would be sufficiently for the underlying factor (Brown, 2006). Next, the resulting factor structure was further tested by CFA using AMOS version 24.0. To test if the factor structure fit well of the sample data, we examined the following fit indices: 1$)$ chi-square per degree of freedom $\left(\chi^{2} / \mathrm{df}\right)<5$ (Schumacker \& Lomax, 
2010), 2) the values of Incremental Fit index (IFI) Comparative fit index (CFI) and Tucker-Lewis Index (TLI) $>0.90$ (acceptable fit) and 0.95 (excellent fit); and 3) RMSEA < 0.05 and 0.08 for close and reasonable fit, respectively (Byrne, 2009; Hu \& Bentler, 1999; Marsh, Hau, \& Wen, 2004). Finally, correlations among the PWB-S and external variables were performed to test the convergent validity of the scale.

\subsection{Ethical Consideration}

The Research Ethics Committee of a University in Thailand approved the study protocols conducted in a university and secondary schools in Thailand. The Research Ethics Committee of a University in Singapore approved the study protocol for the university sample in Singapore. After receiving verbal and written information about the study objectives and procedures from the researchers, participants were asked to sign the informed consent to participate and complete the self-reported questionnaire. The researchers emphasized the issues of voluntary participation and confidentiality whereby participants' personal information were not placed on their questionnaire.

\section{Results}

\subsection{Descriptions of Participants' Characteristics}

Totally, Sample 1, 2, 3 and 4 encompassed 966, 695, 624, and 673 participants respectively (Table 1). All Samples contained more female and male participants. Regarding age, Sample 4 contained students had the average age higher

Table 1. Characteristics of study participants.

\begin{tabular}{|c|c|c|c|c|c|c|c|c|}
\hline & \multicolumn{2}{|c|}{ Sample $1(n=966)$} & \multicolumn{2}{|c|}{ Sample $2(n=695)$} & \multicolumn{2}{|c|}{ Sample $3(n=624)$} & \multicolumn{2}{|c|}{ Sample $4(n=673)$} \\
\hline & $\mathbf{n}$ & $\%$ & $\mathbf{n}$ & $\%$ & $\mathrm{n}$ & $\%$ & $\mathrm{n}$ & $\%$ \\
\hline \multicolumn{9}{|l|}{ Gender } \\
\hline Male & 307 & 31.80 & 217 & 31.20 & 265 & 42.50 & 193 & 27.70 \\
\hline Female & 650 & 67.30 & 473 & 68.10 & 359 & 57.50 & 411 & 59.10 \\
\hline Missing & 9 & 0.90 & 5 & 0.70 & - & - & 92 & 13.20 \\
\hline \multicolumn{9}{|l|}{ Religion } \\
\hline Buddhism & 913 & 94.50 & 649 & 93.40 & 574 & 92.00 & 127 & 18.20 \\
\hline Christian & 20 & 2.10 & 18 & 2.60 & 26 & 4.20 & 172 & 24.70 \\
\hline Islam & 13 & 1.30 & 12 & 1.70 & 9 & 1.40 & 55 & 7.90 \\
\hline Others & - & - & 16 & 2.30 & 15 & 2.40 & 342 & 49.20 \\
\hline \multirow[t]{2}{*}{ Missing } & 20 & 2.1 & & & & & & \\
\hline & Mean & SD & Mean & SD & Mean & SD & Mean & SD \\
\hline Age & 20.21 & 1.51 & 20.34 & 1.43 & 16.14 & 0.97 & 22.39 & 5.18 \\
\hline
\end{tabular}

Note: Sample 1 = University students, Thailand Time 1, Sample $2=$ University students, Thailand Time 2 Sample 3 = Secondary school students, Thailand, Sample 4 = University students, Singapore. 
than other samples whereas Sample 3 had the lowest average. This is reasonable as Sample 3 included younger students from secondary school in Thailand. Concerning religion, Sample 1, 2, and 3 were mostly Buddhist whilst most undergraduate students in Sample 4 were identified with other religions.

\subsection{Factor Structure of the PWB: Exploratory Factor Analyses}

Factor analyses indicated that the 18-item PWB-S had two factors across the four samples (Table 2) and almost all items loaded strongly and cleanly on their respective factors $(\lambda>0.32)$. However, three items (item 1,7 and 17) had weak loadings, suggesting that they might not capture the underlying construct (PWB). Therefore, they were excluded from further analyses. Item 1 ("Influenced by people with strong opinion") and item 17 ("Have done all things in life") had weak loadings across the four sample whereas item 7 ("confidence in my opinions, even if they are contrary to the general consensus") did not work

Table 2. Factor loadings of the PWB Scale across four samples.

\begin{tabular}{|c|c|c|c|c|c|c|c|c|}
\hline \multirow[t]{2}{*}{ Questionnaire Items } & \multicolumn{2}{|c|}{$\begin{array}{c}\text { Sample } 1 \\
(\mathrm{n}=966) \\
\text { Factor Loading }\end{array}$} & \multicolumn{2}{|c|}{$\begin{array}{c}\text { Sample } 2 \\
(\mathrm{n}=695) \\
\text { Factor Loading }\end{array}$} & \multicolumn{2}{|c|}{$\begin{array}{c}\text { Sample } 3 \\
(\mathrm{n}=624) \\
\text { Factor Loading }\end{array}$} & \multicolumn{2}{|c|}{$\begin{array}{c}\text { Sample } 4 \\
(\mathrm{n}=673) \\
\text { Factor Loading }\end{array}$} \\
\hline & F1 & F2 & F1 & F2 & F1 & F2 & F1 & F2 \\
\hline 13. Judge self by what one thinks as important, not by others & 0.71 & 0.03 & 0.74 & 0.10 & 0.60 & -0.06 & 0.52 & 0.08 \\
\hline 9. Life as a process of learning, changing and growth. & 0.69 & 0.10 & 0.69 & 0.12 & 0.52 & 0.09 & 0.53 & -0.05 \\
\hline 12. Like one's personality. & 0.66 & 0.16 & 0.64 & 0.21 & 0.63 & 0.12 & 0.60 & -0.14 \\
\hline 14. Good at managing responsibilities and daily life. & 0.65 & 0.19 & 0.66 & 0.25 & 0.63 & & 0.64 & -0.02 \\
\hline 2. Feel in charge of life situations. & 0.64 & 0.09 & 0.69 & 0.16 & 0.65 & 0.11 & 0.57 & -0.10 \\
\hline 11. Never wander aimlessly through life. & 0.63 & 0.17 & 0.59 & 0.26 & 0.60 & 0.13 & 0.59 & -0.07 \\
\hline 3. Important to have new experiences to challenge self. & 0.59 & 0.02 & 0.69 & 0.09 & 0.62 & -0.06 & 0.43 & 0.07 \\
\hline 6. Pleased with how things turned out in life. & 0.52 & 0.19 & 0.61 & 0.17 & 0.44 & & 0.51 & -0.26 \\
\hline 10. Perceived by others as a giving person. & 0.49 & 0.14 & 0.50 & 0.15 & 0.44 & & 0.28 & -0.12 \\
\hline 7. Confidence in one's opinions. & 0.40 & -0.21 & 0.50 & -0.29 & 0.30 & -0.29 & 0.65 & 0.09 \\
\hline 17. Have done all things in life. & 0.16 & -0.15 & 0.24 & -0.03 & 0.10 & -0.19 & -0.08 & 0.36 \\
\hline 4. Difficulty in Maintaining close relationships & 0.13 & 0.63 & 0.13 & 0.67 & 0.08 & 0.54 & -0.06 & 0.65 \\
\hline 18. Disappointed about one's life achievements. & 0.09 & 0.56 & 0.18 & 0.61 & 0.23 & 0.47 & 0.31 & 0.48 \\
\hline 16. Not many experiences with warm and trusting relationships. & 0.18 & 0.53 & 0.26 & 0.57 & 0.17 & 0.54 & 0.11 & 0.82 \\
\hline 8. Daily life demand often make one down. & 0.03 & 0.53 & 0.05 & 0.68 & 0.15 & 0.52 & 0.16 & 0.47 \\
\hline 15. Give up making life improvements or changes. & 0.19 & 0.52 & 0.15 & 0.63 & 0.24 & 0.47 & 0.12 & 0.41 \\
\hline 5. Live one day at a time and without thinking about the future. & 0.23 & 0.43 & 0.19 & 0.52 & 0.27 & 0.43 & 0.23 & -0.19 \\
\hline 1. Influenced by people with strong opinions. & -0.09 & 0.197 & -0.15 & 0.28 & -0.03 & 0.16 & 0.19 & -0.06 \\
\hline Cronbach's alpha & 0.85 & 0.70 & 0.82 & 0.74 & 0.81 & 0.68 & 0.83 & 0.56 \\
\hline
\end{tabular}

Note: Sample 1 = University students, Thailand Time 1, Sample 2 = University students, Thailand Time 2, Sample $3=$ Secondary school students, Thailand, Sample 4 = University students, Singapore. 
well only on the sample 3 .

After reviewing the content of the items, we named factors of the PWB as the Autonomy \& Growth and the Negative Triad factors. For the sample 1, 2, and 4, the Autonomy \& Growth factor contained 10 items $(2,3,6,7,9,10,11,12,13$ and 14) whereas the sample 3 had only 9 items (with the aforementioned items except item 7). The Negative Triad entailed 6 items across the four samples. However, the sample 1, 2, and 3 had items 4, 5, 8, 15, 16, and 18 whereas the sample 4 had slightly different items $(4,8,15,16,17$ and 18). In other words, item 5 was removed from the sample 4 and was replaced by item 17 .

Results from reliability analyses suggested that factors for the sample 1 and 2 had acceptable internal consistency reliability as evidenced by Cronbach's alpha (a) $>0.70$ (Table 2). However, for sample 3 and 4, the negative triad factor had slightly low reliability, with Cronbach's alpha ( $\alpha$ ) was 0.68 and 0.56 respectively.

\subsection{Confirmed Structure of the PWB: Confirmatory Factor Analyses}

The resulting two-factor structures of the PWB were submitted to IBM AMOS version 24. Figure 1 and Figure 2 portrayed results for sample 1, 2, 3 and 4 respectively. All samples showed acceptable fit indices, suggesting that the two-factor PWB-S fit well with the sample data. Sample 1 and 2 contained the same questionnaire items with the Autonomy \& Growth (10 items) and Negative Triad ( 6 items) factors. These items were also the same as those reported in EFA. All factor loadings achieved statistical significance $(p<0.001)$ with the range of
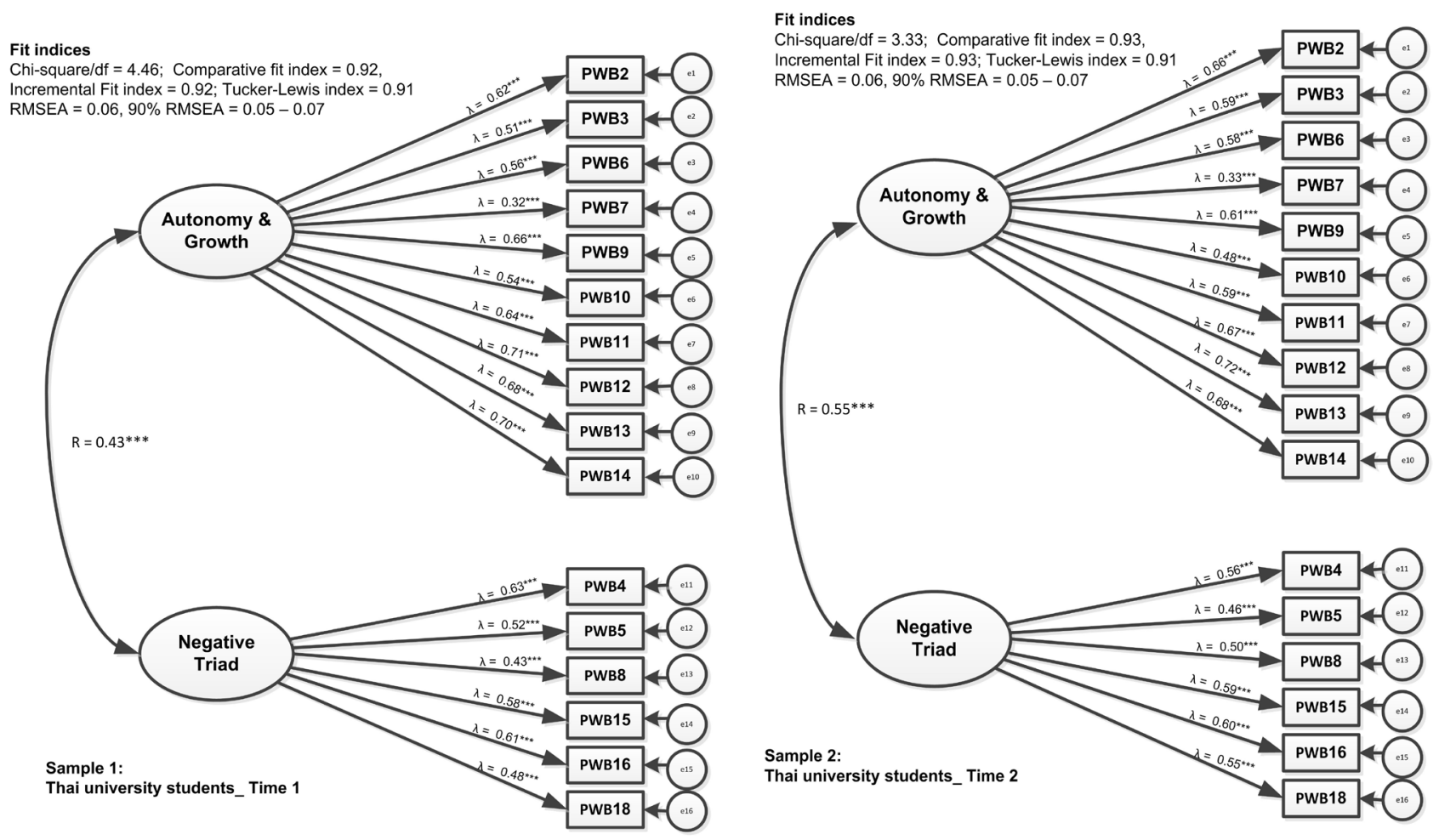

Figure 1. Factor structure of psychological well-being scale for sample 1 and sample 2. 


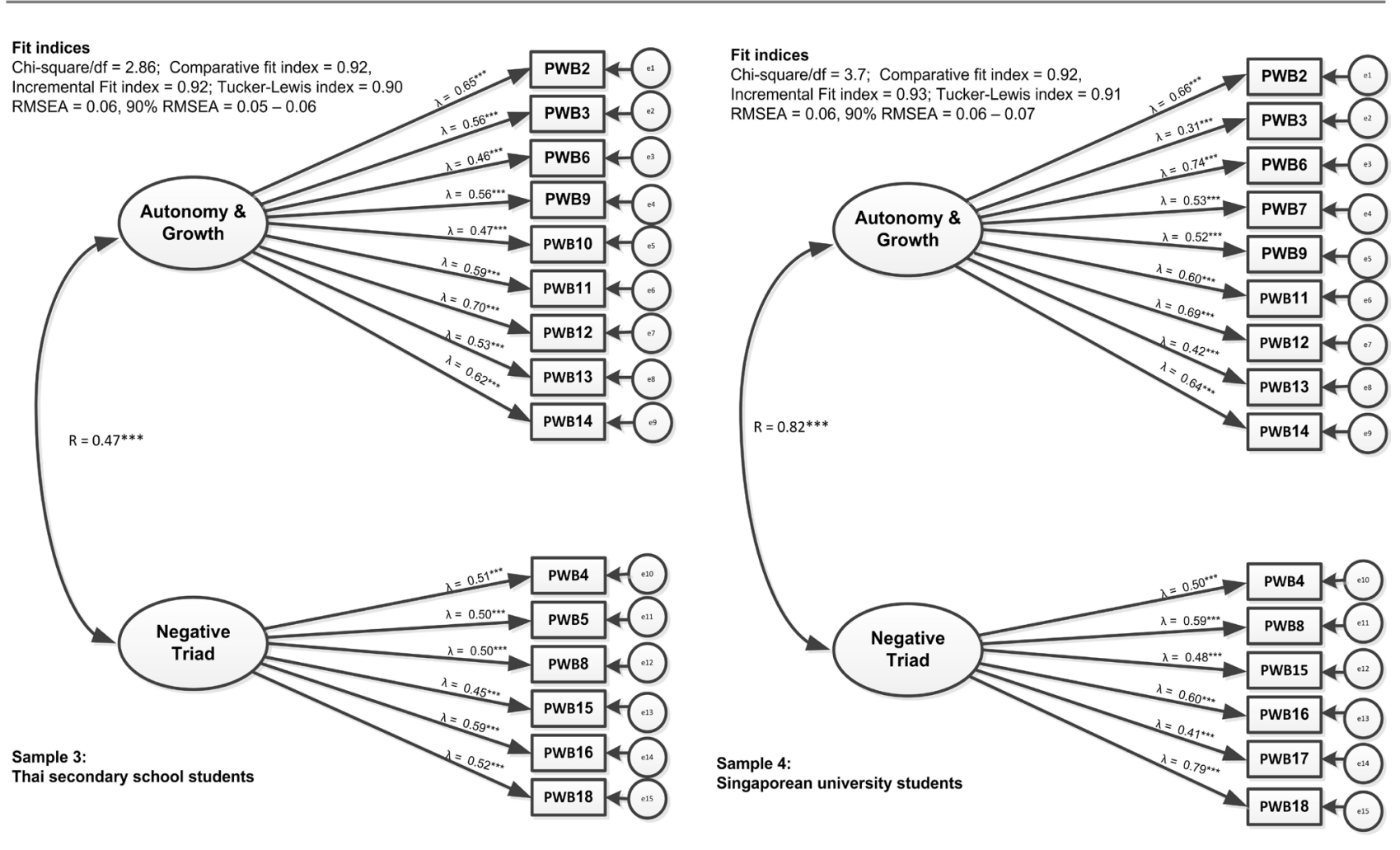

Figure 2. Factor structure of psychological well-being scale for sample 3 and sample 4.

0.50 - 0.72. Factor loadings for the sample 1 and 2 were comparable across all items.

Sample 3 entailed the same items as those in the sample 1 and 2 except that item 7 was removed from the Autonomy \& Growth factor due to low factor loading $(\lambda<0.30)$ (Figure 2$)$. The remaining items had factor loadings with statistical significance $(p<0.001)$ ranging from 0.50 to 0.70 . The magnitude of the factor loadings was comparable to those in Sample 1 and 2, except for item 13 ("Important to have new experiences to challenge self"). Specifically, item 13 had $\lambda=0.72,0.70$, and 0.53 for the sample 1,2 , and 3 respectively.

Finally, items in the sample 4 were almost the same as those in the sample 1 and 2 except that item 5 ("Live one day at a time and without thinking about future") was replaced by item 17 ("Have done all things in life"). The remaining items had factor loadings with statistical significance $(p<0.001)$ ranging from 0.31 to 0.79 . In general, items in the sample 4 had comparable factor loadings with those in sample 1 and 2, except items 3 ("importance to have new experiences in life), 6 ("Pleased with how things turned out in life") and 18 ("Disappointed about life achievement"). Specifically, item 3 had noticeable lower loading and item 6 and 18 had higher loadings.

\subsection{Relationships with External Variables}

Table 3 suggested that the Autonomy \& Growth factor had significant positive correlations with support from others, support from family, and support from friends across four samples. Similarly, the Negative Triad factors had significant 
Table 3. Correlation among study variables across four samples.

\begin{tabular}{ccccccccc}
\hline \multirow{2}{*}{$\begin{array}{c}\text { Independent } \\
\text { Variables }\end{array}$} & \multicolumn{3}{c}{ The Autonomy \& Growth Factor $(\mathrm{r})$} & \multicolumn{4}{c}{ The Negative Triad Factor $(\mathrm{r})$} \\
\cline { 2 - 9 } & $\begin{array}{c}\text { Sample } \\
1\end{array}$ & Sample & Sample & Sample & Sample & Sample & Sample & Sample \\
& 2 & 3 & 4 & 1 & 2 & 3 & 4 \\
\hline Support from others & $0.39^{* *}$ & $0.33^{* *}$ & $0.27^{* *}$ & $0.30^{* *}$ & $0.30^{* *}$ & $0.33^{* *}$ & $0.31^{* *}$ & $0.40^{* *}$ \\
Support from family & $0.38^{* *}$ & $0.34^{* *}$ & $0.26^{* *}$ & $0.34^{* *}$ & $0.28^{* *}$ & $0.35^{* *}$ & $0.35^{* *}$ & $0.33^{* *}$ \\
Support from friends & $0.33^{* *}$ & $0.32^{* *}$ & $0.31^{* *}$ & $0.34^{* *}$ & $0.29^{* *}$ & $0.31^{* *}$ & $0.33^{* *}$ & $0.50^{* *}$ \\
\hline
\end{tabular}

Note: Sample $1=$ Thai university Time 1; Sample 2 = Thai university Time 2; Sample 3 = Thai Secondary school students; Sample $4=$ Singaporean University students. $r=$ correlation coefficients. ${ }^{\star}$ Significant level at $\alpha=0.05 ;{ }^{*}$ Significant level at $\alpha=0.01$

correlations with all social support subscales across all samples. This evidence further supported the convergent validity of the PWB-S.

\section{Discussion}

The aim of this study was to test the psychometric properties of the Ryff's Psychological Well-being scale (PWB-S) using large four data sets across two cultures and time. The four samples include: Thai university students Time 1 ( $\mathrm{n}=$ 966), Thai university students Time $2(\mathrm{n}=695)$, Thai high school students $(\mathrm{n}=$ $624)$ and university students in Singapore $(n=673)$. It is important to understand the measurement characteristics of the PWB-S as it is widely used and cited. Ryff \& Keyes (1995) provided empirical support for the six-dimension of PWB which supported by another study in Western country (Clarke et al., 2001). However, to our knowledge, this is the first cross-cultural study to test the factorial structure of the PWB-S across assessment points (Time 1 and 2), countries (Thailand and Singapore), languages (Thai and English version) and data collection methods (paper-and-pencil questionnaire and online questionnaire). Findings in this study revealed that most PWB-S items showed acceptable internal consistency reliability. In contrast to previous studies, our results testified that the PWB-S had two distinct constructs: The Autonomy \& Growth and Negative Triad.

The Autonomy \& Growth factor of PWB-S contained similar questionnaire items across the four samples. Sample 1, 2, and 4 had 10 identical items with slightly different factor loadings. Sample 3 had 9 items as one of which ("Confidence in one opinion") was removed due to a low factor loading. This is not surprising given that Thai secondary school students (sample 3) were younger, less independent and less mature than other samples and thus "the confidence in their opinion" might not be a strong aspect of their psychological well-being. In general, the contents regarding Autonomy described that individuals: judged themselves by their own thought (not by others), felt confidence in their own opinion, liked their personality, perceived by others as a giving person, felt in charges of life situations, and was good at managing responsibilities and daily life. The contents concerning Growth addressed that persons: felt that life as a process of learning, changing and growth, were pleased with how things turned 
out in their life, had never wandered aimlessly through life, and accepted the importance of having new experiences to challenge self. We think that all items clustered together well and make good theoretical/conceptual senses.

Autonomy is one of the central concepts in the self-determining theory (SDT, Deci \& Ryan, 2000). Autonomy refers to regulation by oneself and reflects selfendorsed behavior, self-reliance and independence (Deci \& Ryan, 2000). A previous research tested and reported a sound instrument, the Autonomy-Connectedness scale (ACS; Bekker \& Van Asssen, 2006). This scale contains such items as: not being occupied with others'feelings/experiences, putting aside other people's comments/opinions, feeling at ease quickly when facing new situations and new environments, being adventurous, being to manage problems of my own, among others (Bekker \& Van Asssen, 2006). Our findings are consistent with conceptualization of the SDT concerning autonomy. The PWB-S Items concerning autonomy were also similar to contents in the ACS scale.

The Negative Triad factor of the PWB-S contained identical questionnaire items (6 items) across Sample 1, 2, and 3 with slightly different factor loadings. Sample 4 (Singaporean university students) also had 6 items but one included item (Have done all things life) was different from other samples. Such finding might reflect culture differences between Thai and Singapore. Specifically, the university environment in Singapore is perceived to be more stressful and competitive than that in Thailand. To be successful, Singaporean undergraduate students are required to perform extremely well and they have to know that they have done the best. Therefore, it is not surprising that "Have done all things in life" was part of their psychological well-being. In general, the contents of the Negative Triad factor delineate how individuals: felt disappointed about their achievement, felt that daily life demands make them down, perceived difficulty in maintaining relationships with others, did not have warm and trusting relationships, gave up to make life improvements or changes and lived without thinking about the future. Such items reflect negative perception toward self, others, and future. Students with low scores on the above-mentioned items (lower Negative Triad) would reflect greater psychological well-being.

Cognitive triad is one of the main concepts in the Beck's cognitive theory of depression (Beck, Rush, Shaw, \& Emery, 1987). Cognitive triad indicates how individuals have the tendency to hold negative views of themselves, the world and the future. Those people are prone to experience mental problems, including depression. The cognitive triad inventory (CTI) had been widely used to measure cognitive triad (Beckham, Leber, Boyer \& Cook, 1986). Examples of the CTI items include: Being a failure and inadequate, facing with many difficulty, feeling that most people are not useful and friendly, having messed up with all meaningful relationships, thinking that things will not work well in the future, and having no reason to be hopeful about the future (Beckham et al., 1986). Conceptually, the Negative Triad factor in our study was in line with cognitive triad in Beck's theory of depression and questionnaire items in the Negative 
Triad factor were similar to those in the CTI.

Our findings contradict with the exiting evidence. Previous studies evaluated the construct validity of 18-item PWB-S and reported the six-factor structure on three large databases of American adults and a large Canadian database (Clark et al., 2001; Hsu et al., 2017; Ryff \& Keyes, 1995; Springer \& Hauser, 2006). The factors included autonomy, environmental mastery, personal growth, purpose in life, relations with others, and self-acceptance. However, Ryff \& Keyes (1995) found high correlations among the six factors with the largest correlation between the environmental mastery and the self-acceptance factor $(r=0.85)$. Such high correlations indicated conceptual overlap among the underlying constructs. Clark et al. (2001) also found that four items might measure more than two constructs, again highlighting the conceptual overlap. Furthermore, Springer \& Hauser (2006) reported large correlations among the six factors with the largest one was greater than 0.90 . For our study, it might be possible that large correlations existed among questionnaire items and; therefore, they clustered into two factors (rather than six). Another possible explanation could be that four samples in our research were younger than those in previous studies. Furthermore, cultural differences might play a role whereby greater Autonomy \& Growth and lower Negative Triad are critical components of psychological well-being among younger people in Asian cultures.

Convergent validity of the two factors are supported by their significant relationships with all external variables (three social support subscales) across four samples. Specifically, students who had greater support from family, friends and significant others reported higher Autonomy \& Growth levels. Similarly, those who received more social support tended to experience lower levels of Negative Triad. Note that the highest scores signified the lowest level of Negative Triad.

\section{Strengths and Limitations}

This study was strengthened by the use of CFA (with AMOS), which provided a sophisticated way to examine the construct validity of the PWB-S using goodness-of-fit indices and thus enhancing accuracy in estimating crucial parameters (i.e., factor loadings and correlation coefficients). In EFA, we explored different factor structures $(2,3,4,5$, and 6 factors) to determine the best solution taken both statistical (such as Eigen value) and substantive (such as face validity and parsimony) aspects into consideration. Furthermore, the large sample size (966, 695,624 , and 673 students) might increase the generalizability of research findings. In addition, our findings maintained across two assessment points (Time 1 and 2), cultures (Thai and Singapore), data collection tools (online and hardcopy questionnaires) and languages (Thai and English). Thus, internal validity and generalizability were strengthened. Besides, this is the first validation study of the PWB-S in Thai version and we think that this short and simple instrument requires little time to complete and it is thus efficiently administered. For that reason, it may be a suitable instrument for clinical use in community stu- 
dies. Nonetheless, this study also has some limitations in that convenience sampling was utilized. The samples included only one university in Thailand and one in Singapore; and three Thai secondary schools in Thailand. Therefore, our results may not be generalized to youths in other settings or the general populations.

\section{Conclusion}

In summary, the Ryff's Psychological Well-being scale (PWB-S) in both Thai and English versions had sound psychometric properties (construct validity, convergent validity and reliability). This study has implications to clinical practice and future research. Specifically, the Thai-version scale can be used to measure psychological well-being among youths (at Secondary schools and University levels). Similarly, the English-version PWB-S can be used by to assess psychological well-being among younger people in Singapore and probably in other English-speaking countries. Psychosocial interventions can be offered to the study participants according to their levels of PWB (such as maintaining, promoting and/or enhancing the well-being). The scale can be used to advance future quantitative research such as investigating the effectiveness of interventions on PWB, examining the predicting effect of $\mathrm{PWB}$ on health-related outcomes, and testing psychometric properties of the PWB-S on other populations. To strengthen external validity of research findings, multi-centered recruitments with large sample sizes and cross-cultural studies are also encouraged.

\section{Conflicts of Interest}

The authors declare no conflicts of interest regarding the publication of this paper.

\section{References}

Abbott, R. A., Ploubidis, G. B., Huppert, F. A., Kuh, D., Wadsworth, M. E., \& Croudace, T. J. (2006). Psychometric Evaluation and Predictive Validity of Ryff's Psychological Well-Being Items in a UK Birth Cohort Sample of Women. Health and Quality of Life Outcome, 4, Article No. 76. https://doi.org/10.1186/1477-7525-4-76

Beck, A. T., Rush, A. J., Shaw, B. F., \& Emery, G. (1987). Cognitive Therapy of Depression. New York: Guilford Press.

Bekker, M. H. J., \& Van Assen, M. A. L. M. (2006). A Short Form of the Autonomy Scale: Properties of the Autonomy-Connectedness Scale (ACS-30). Journal of Personality Assessment, 86, 51-60. https://doi.org/10.1207/s15327752jpa8601_07

Brislin, R. W. (1986). The Wording and Translation of Research Instruments. In W. L. Lonner, \& J. W. Berry (Eds.), Cross-Cultural Research and Methodology Series, Vol. 8. Field Methods in Cross-Cultural Research (pp. 137-164). Thousand Oaks, CA: Sage Publications.

Brown, T. A. (2006). Confirmatory Factor Analysis for Applied Research. New York: Guilford Press.

Byrne, B. M. (2009). Structural Equation Modeling with AMOS: Basic Concepts, Applications, and Programming (2nd ed.). New York: Routledge. 
Clarke, P. J., Marshall, V. M., Ryff, C. D., \& Wheaton, B. (2001). Measuring Psychological Well-Being in the Canadian Study of Health and Aging. International Psychogeriatrics, 13, 79-90. https://doi.org/10.1017/S1041610202008013

Deci, E., \& Ryan, R. (2000). The "What" and "Why" of Goal Pursuits: Human Needs and the Self-Determination of Behavior. Psychological Inquiry, 11, 227-268. https://doi.org/10.1207/S15327965PLI1104_01

Diener, E. (1984). Subjective Well-Being. Psychological Bulletin, 95, 542-575. https://doi.org/10.1037/0033-2909.95.3.542

Fabrigar, L. R., MacCallum, R. C., Wegener, D. T., \& Strahan, E. J. (1999). Evaluating the Use of Exploratory Factor Analysis in Psychological Research. Psychological Methods, 4, 272-299. https://doi.org/10.1037/1082-989X.4.3.272

Faudzi, F. N. M., Armitage, C. J., Bryant, B., \& Brown, L. J. E. (2018). Cultural Adaptations to the Measurement of Attitudes to Aging: Psychometric Assessment of the Malay Reaction of Aging Questionnaire. Assessment, 1-13. https://doi.org/10.1177/1073191118766400

Grove, S. K., Burns, N., \& Gray, J. R. (2013). The Practice of Nursing Research: Appraisal, Synthesis, and Generation of Evidence (7th ed.). St. Louis, MO: Elsevier.

Hernandez, R., Bassett, S. M., Boughton, S. W., Schuette, S. A., Shiu, E. W., \& Moskowitz, J. T. (2018). Psychological Well-Being and Physical Health: Associations, Mechanisms, and Future Directions. Emotional Review, 10, 18-29.

https://doi.org/10.1177/1754073917697824

Hsu, H. Y., Hsu, T. L., \& Lee, K. (2017). Evaluating the Construct Validity of Ryffs Scales of Psychological Well-Being Using Exploratory Structural Equation Modeling. Journal of Psychological Assessment, 35, 633-638. https://doi.org/10.1177/0734282916652756

Hu, L.-T., \& Bentler, P. M. (1999). Cutoff Criteria for Fit Indexes in Covariance Structure Analysis: Conventional Criteria versus New Alternatives. Structural Equation Modeling, 6, 1-55. https://doi.org/10.1080/10705519909540118

Kafka, G. J., \& Kozma, A. (2002). The Construct Validity of Ryff's Scales of Psychological Well-Being (SPWB) and Their Relationship to Measures of Subjective Well-Being. Social Indicators Research, 57, 171-190. https://doi.org/10.1023/A:1014451725204

Keyes, C. L. M., Shmotkin, D., \& Ryff, C. D. (2002). Optimizing Well-Being: The Empirical Encounter of Two Traditions. Journal of Personality and Social Psychology, 82, 1007-1022. https://doi.org/10.1037/0022-3514.82.6.1007

Liu, Q., Shono, M., \& Kitamura, T. (2009). Psychological Well-Being, Depression and Anxiety in Japanese University Students. Depression and Anxiety, 26, E99-E105. https://doi.org/10.1002/da.20455

Marmot, M., Ryff, C. D., Bumpass, L. L., Shipley, M., \& Marks, N. F. (1997). Social Inequalities in Health: Next Questions and Converging Evidence. Social Science \& Medicine, 44, 901-910. https://doi.org/10.1016/S0277-9536(96)00194-3

Marsh, H. W., Hau, K.-T., \& Wen, Z. (2004). In Search of Golden Rules: Comment on Hypothesis Testing Approaches to Setting Cut off Values for Fit Indexes and Dangers in Overgeneralizing Hu and Bentler's (1999) Findings. Structural Equation Modeling, 11,320-341. https://doi.org/10.1207/s15328007sem1103_2

Paperny, D. M. (2011). Handbook of Adolescent Medicine and Health Promotion. Singapore: World Scientific. https://doi.org/10.1142/7860

Ramkisson, S., Pillay, B. J., \& Sartorius, B. (2016). Anxiety, Depression and Psychological Well-Being in a Cohort of South African Adults with Type 2 Diabetes. South African Journal of Psychiatry, 22, a359. https://doi.org/10.4102/sajpsychiatry.v22i1.935 
Ryan, R. M., \& Deci, E. L. (2001). On Happiness and Human Potentials: A Review of Research on Hedonic and Eudaimonic Well-Being. Annual Review of Psychology, 52, 141-166. https://doi.org/10.1146/annurev.psych.52.1.141

Ryff, C. D. (1989a). In the Eye of the Beholder: Views of Psychological Well-Being among Middle-Aged and Older Adults. Psychology and Aging, 4, 195-210.

https://doi.org/10.1037/0882-7974.4.2.195

Ryff, C. D. (1989b). Happiness Is Everything, or Is It? Explorations on the Meaning of Psychological Well-Being. Journal of Personality and Social Psychology, 57, 1069-1081. https://doi.org/10.1037/0022-3514.57.6.1069

Ryff, C. D. (1995). Psychological Well-Being in Adult Life. Current Directions in Psychological Science, 4, 99-104. https://doi.org/10.1111/1467-8721.ep10772395

Ryff, C. D. (2014). Psychological Well-Being Revisited: Advances in the Science and Practice of Eudaimonia. Psychotherapy and Psychosomatics, 83, 10-28.

https://doi.org/10.1159/000353263

Ryff, C. D., \& Keyes, C. M. (1995). The Structure of Psychological Well-Being Revisited. Journal of Personality and Social Psychology, 69, 719-727. https://doi.org/10.1037/0022-3514.69.4.719

Ryff, C. D., \& Singer, B. (1996). Psychological Well-Being: Meaning, Measurement, and Implications for Psychotherapy Research. Psychotherapy and Psychosomatics, 65, 14-23. https://doi.org/10.1159/000289026

Ryff, C. D., \& Singer, B. H. (2006). Best News Yet on the Six-Factor Model of Well-Being. Social Science Research, 35, 1103-1119. https://doi.org/10.1016/j.ssresearch.2006.01.002

Ryff, C. D., Keyes, C. L., \& Hughes, D. L. (2003). Status Inequalities, Perceived Discrimination, and Eudaimonic Wellbeing: Do the Challenges of Minority Life Hone Purpose and Growth? Journal of Health and Social Behavior, 44, 275-291. https://doi.org/10.2307/1519779

Ryff, C. D., Lee, Y. H., Essex, M. J., \& Schmutte, P. S. (1994). My Children and Me: Mid-Life Evaluations of Grown Children and of Self. Psychology and Aging, 9, 195-205. https://doi.org/10.1037/0882-7974.9.2.195

Ryff, C. D., Singer, B., \& Love, G. D. (2004). Positive Health: Connecting Well-Being with Biology. Philosophical Transactions of the Royal Society of London. Series B: Biological Sciences, 359, 1383-1394. https://doi.org/10.1098/rstb.2004.1521

Schumacker, R. E., \& Lomax, R. G. (2010). A Beginner's Guide to Structural Equation Modeling (3rd ed.). New York: Routledge.

Springer, K. W., \& Hauser, R. M. (2006). An Assessment of the Construct Validity of Ryff's Scales of Psychological Well-Being: Method, Mode and Measurement Effects. Social Science Research, 35, 1080-1102. https://doi.org/10.1016/j.ssresearch.2005.07.004

Van Dierendonck, D. (2004). The Construct Validity of Ryff's Scales of Psychological Well-Being and Its Extension with Spiritual Well-Being. Personality and Individual Differences, 36, 629-643. https://doi.org/10.1016/S0191-8869(03)00122-3

World Health Organization (WHO) (2011). Adolescence Development. Resource Document.

http://www.who.int/maternal_child_adolescent/topics/adolescence/dev/en/index.html

World Health Organization (WHO) (2014). Recognizing Adolescence. http://apps.who.int/adolescent/second-decade/section2/page1/recognizing-adolescence html

Zimet, G. D., Dahlem, N. W., Zimet, S. G., \& Farley, G. K. (1988). The Multidimensional Scale of Perceived Social Support. Journal of Personality Assessment, 52, 30-41.

https://doi.org/10.1207/s15327752jpa5201_2 